directly or deduced indirectly from their vapour pressures, and the relation connecting the osmotic and vapour pressures is quite independent of the "gas laws holding for solutions."

Leaving out of consideration the experiments made before the solutions were stirred-for on Prof. Kahlenberg's own showing these are not good-his conclusion that the gas laws do not hold for dilute solutions in pyridine is based on four experiments. If one may take No. 59 (p. 201) as a type of these, it is easy to show that the experiment is valueless.

The sugar solution used is $0.125 \mathrm{grm}$. mol. per litre, and a pressure of $98 \mathrm{~cm}$. of mercury is reached, but the theoretical value is some 3 atmos. Now on p. 184 the diameter of his gauge is given as $0.5 \mathrm{~mm}$., and he says that at the end of three days $0.115 \mathrm{grm}$. sugar has come through the membrane-this quantity represents 2.8 c.c. of solution. If we assume that this volume of solution came through the membrane at a uniform rate, a simple calculation will show that the rate is equivalent to a fall of $20 \mathrm{~cm}$. per hour in the gauge. No wonder the theoretical pressure was never reached!

Foxcombe, near Oxford.

BERKELEY

E. G. J. Hartley.

Diurnal Variation of the Ionisation in Closed Vessels. IN his letter on this subject published in NaTure of May 3 (p. 8) Mr. G. C. Simpson is, I venture to think, under a misapprehension regarding the conditions which determine the variations of the earth's electric field. His statement of the problem, which I have slightly abbreviated, is as follows:- "It is usual to accept that there is a negative charge on the earth's surface, and the corresponding positive charge is a volume charge distributed in the atmosphere. There is very little volume charge in the air close to the earth's surface, so the relation between potential gradient and charge on the earth's surface is given by $d v / d h=-4 \pi \sigma$. Hence it follows that with a given charge on the earth's surface and the corresponding charge in the atmosphere above, the vertical distribution of the charge and the conducting state of the upper atmosphere do not in the slightest affect the potential gradient within a few metres of the earth's surface."

All this is very true, but it is equally true that with a constant charge on the earth's surface nothing whatever will affect the potential gradient close to it. Since the potential gradient is a constant multiple of the surface density, it is absurd to consider the variation of the one whilst the other is kept constan1. The only assumption that it seems safe to make about the state of the earth's surface is that, owing to the relatively high conductivity of the earth's crust, for purposes of atmospheric electricity it may be treated as an equipotential surface. The charge in any particular region will be determined by the distribution of electrification and ionisation in the atmosphere, and will readjust itself almost instantaneously when any change takes place in the external conditions. It will not, as an incautious reader might gather from Mr. Simpson's letter, behave as if it were glued to the surface of the earth.

In my letter of April 22 I illustrated my point by considering the analogy with the case of ionised air between two parallel plates maintained at a constant difference of potential. As this comparison is inaccurate, I shall take the liberty of putting the case in another way, in the hope that it may prove more convincing. The earth is to be regarded as a conducting sphere which is continuously receiving a negative charge in certain areas-probably those in which rain is falling-and losing it again by conduction through the atmosphere from all the rest of its surface. Since the observations on the earth's field only refer to fine-weather regions, we need only consider what happens over them. There will be an earth-air current which, under specified conditions, will have attained a steady value, the charge on the earth's surface being that required to give the necessary potential gradient to drive the current. Suppose that by some means the ionisation at some distance from the surface suffers a permanent increase locally, whilst the air close to the surface is unaffected. It is clear that, whrtever view is taken of the distribution of the charges producing the earth's field, the increase in the ionisation will produce a local increase in the earth-air current; but by hypothesis the conductivity of the air close to the earth is unaltered, so that the increase in the current must be accompanied by an increase in the potential gradient close to the surface. This is, of course, produced by negative electricity flowing from other parts of the earth.

The above, I imagine, is an exaggerated but otherwise trustworthy picture of the effect an ionising radiation from outside would have on the earth's electric field. The conductivity produced by the rays in the upper atmosphere must be enormous compared with the effects close to the earth. Even if the rays were homogeneous, only a mere trace would remain after passing through a layer of air roughly equivalent in absorbing power to $76 \mathrm{~cm}$. of mercury. But it is far from probable that they are homogeneous, and any want of homogeneity would exaggerate the effect. Other factors conspire to this end: the presence of dust near the earth loading the ions and the smaller rate of recombination at low pressures; whilst the increase in the mobility of the ions at low pressures would just compensate for the feebler absorbing power of the upper atmosphere.

It will be observed that the effect on the earth's field of an increase in the ionisation of the atmosphere depends entirely on where that increase takes place. If the conductivity increases in a greater ratio close to the earth's surface than it does further away, the result ought to be a fall in the potential gradient. Mr. Simpson rightly points out that such a relation between the potential gradient and the leakage of electricity near the earth's surface has been shown to exist. From my point of view this indicates that the bulk of the ionisation near the earth's surface is not caused by radiation from an external source.

Trinity College, Cambridge, May 12.

O. W. RichaRdson.

\section{Defects in Ostrich Feathers in South Africa}

THE domestication of the ostrich on a practical basis was undertaken in Cape Colony about 1867, and since then ostrich farming has become one of the most important industries in the eastern province. The census of 1904 gave $357,970^{1}$ tame ostriches in the colony, while the export of feathers reached $470,38 \mathrm{r}$ pounds, practically the whole of which came from tame birds; the estimated value of the feathers was $1,058,988 l$., giving about $3 l$. ros. per bird of feather-producing age. During the forty years of domestication the instincts of the ostrich have apparently undergone no change, though its habits are much altered. The feathers cut from the tame bird are shorter, weaker, and not so fluffy as those taken from wild birds, but probably these differences are to be correlated with the greater frequency of plucking, and not with any constitutional change resulting from domestication.

Within recent years much concern has arisen from the prevalence of a defect in the growth of the feather, which seriously reduces the value of the plumes to the farmer. The imperfection, technically known as "barring," takes the form of a series of narrow, chevron-shaped bars or malformations across the whole feather. The general appearance of a moderately affected plume is shown in the accompanying photograph (Fig. I). Examined closely, it is seen that the regularity of the individual barb is much disturbed at the bars, and that the barbules are there defective and only partly differentiated from the barbs. The appearance is such as to suggest that the barbs have been constricted at these particular regions, and that in the development of the feather the barbules have failed to become differentiated and open out, though with a needle their separation can sometimes be effected. Occasionally several barbs will remain joined together at the bars, they also having failed to differentiate. In many cases some of the barbs are shortened, the missing part having broken

1 The statistics are taken from a paper, by the Hon. Arthur Denglass read before the recent meetings of the British Ascociation at Cape Town.
Mr. Douglass is the author of a well-known work, "Ostrich Farming in Mr. Douglass is the author of a well-known work, "Ostrich Farming in
South Africa," and was nne of the pioneers in the domestication of the South Africa," and was ne of the pioneers in the domestication of the
ostrich, and probably the first to hatch the chicks by artificial incubation. ostrich, and probably the first to hatch the chicks by artificial incubation. His death. shortly after the meetings of the Associ

No. I 907, vOL. 74] 